\title{
Burnout and Resiliency Among Neurocritical Care Staff; Potential Solutions to A Growing Problem
}

\author{
Ana-Maria Vranceanu*
}

๑ 2019 Springer Science+Business Media, LLC, part of Springer Nature and Neurocritical Care Society

Over the past decade, there has been a proliferation of research on psychosocial sequelae associated with hospitalization for neurocritical illness for both patients and their informal caregivers (e.g., family and friends who provide unpaid care [1]). We now know that many survivors and their families develop depression, anxiety, and posttraumatic stress [2, 3], due to the sudden and often traumatic onset of the brain injury, as well as the uncertainty of prognosis and recovery trajectory. However, less research has explored the psychological effects of caring for patients and families on neurocritical care staffs' emotional functioning. Burnout is a topic of utmost importance for neurocritical care staff, who are under constant stress due to management of complex medical scenarios, engagement in challenging life or death situations with families, and witnessing death or severe sequelae on a daily basis.

Burnout is a syndrome characterized by emotional exhaustion that can lead to depersonalization and decreased personal accomplishment at work. Emotional exhaustion manifests through feelings of overwhelm and fatigued, inability to face the demands of the job, and to meaningfully engage with others. Over time, a sense of cynical detachment from work can develop, and patients can start to be viewed as objects (e.g., depersonalization). This can be followed by a perception of lack of making meaningful contributions and a feeling of inadequacy at work. Mounting evidence over the past few years has drawn attention to a growing prevalence of burnout among healthcare personnel in a variety of settings, and

\footnotetext{
*Correspondence: avranceanu@mgh.harvard.edu Integrated Brain Health Clinical and Research Program, Department of Psychiatry, Harvard Medical School, One Bowdoin Square, 1st Floor, Suite 100, Boston, MA 02114, USA
}

this has been recently depicted as a threat to healthcare quality and patient safety. This year, the American Heart Association CEO roundtable of 40 members representing more than 10 million employees and families has released "Mental Health: A workforce crises," delineating the problem of workplace burnout and need for interventions. Understanding sources of burnout and developing interventions to decrease it is thus of utmost importance not only for the general wellbeing and health of neurocritical care employees, but also because of its negative effects on patient safety and quality of care, as well as loss of resources due to the high turnover and inability to retain talented staff.

The brief communication by Purvis and colleagues [4] in this issue of Neurocritical Care draws attention to this important yet understudied problem. The authors report on a cross-sectional study of burnout and resiliency (e.g., ability to successfully manage traumatic or highly stressful situations) among neurocritical care staff (75\% nurses). Approximately $45 \%$ of staff members had clinically significant scores on emotional exhaustion and $28 \%$ on depersonalization. Longer time working in the neurocritical care setting was associated with higher emotional exhaustion, and older age was associated with higher resiliency.

While this study has several limitations, including a relatively small sample size comprised primarily of white females, a cross-sectional design, and lack of assessment of important variables such as number of hours worked per week and perceived stress, it provides a starting point toward future research aimed at understanding burnout and resiliency in this population, as well as developing interventions to decrease burnout and increase resiliency. Although levels of resiliency were relatively high in this study, a more in-depth study of resiliency factors

\section{实 Springer}


(e.g., mindfulness, coping and interpersonal support skills) may prove as more valuable for two main reasons. First, resiliency is a dynamic construct that cannot be properly measured with only one scale. Second, understanding which resiliency factors are associated with decreased burnout can help design interventions aimed at directly targeting these exact factors and thus increasing probability of success.

As the authors point out, it will also be important to conduct qualitative interviews with neurocritical care staff to gain an in-depth understanding of the sources of burnout for various categories of staff (e.g., nurses vs. critical care doctors), healthy and unhealthy coping skills, barriers and facilitators, and interest, type, and modality of delivery of a resiliency skills program to decrease burnout and improve resiliency in this population. Developing an intervention aimed at decreasing burnout, albeit critical, is not going to be an easy task, given the time and resources required to deliver or attend such programs within busy neurocritical care settings. While mindfulness-based interventions have shown some promise in decreasing stress and burnout among nurses in a recent systematic review [5], we do lack fully powered randomized controlled efficacy trials. Further, such interventions are not likely to be amenable to implement in busy neurocritical care settings. The ideal intervention would likely need to be brief and teach skills that can be practiced and implemented while at work. Support from neurocritical care leadership is going to be pivotal to allow protected time and encouragement for both participation and skills practice.

While recent research has been focused on decreasing burnout, we should also be focused on prevention of burnout. As Pruvis et al. showed, number of years of work in the neurocritical care setting is associated with increased burnout. Perhaps the most effective and efficient way to address this complex issue is to start with educational information and brief skills training at the time of staff onboarding, before any burnout symptoms develop, and then continue monitoring symptoms and addressing them as needed, with support from leadership. To be successful, we need to develop a culture that supports and models open communication and prioritization of emotional health for both staff, and patients and families.

The neurocritical care field currently lacks preventive interventions for chronic emotional distress both among survivors and families, and among neurocritical care staff. The last decade has led to enormous medical advances, and, as a result, the number of patients surviving hospitalization has dramatically increased. The next decade must bring us novel psychosocial interventions aimed at preventing chronic emotional distress among survivors, their family caregivers, as well as the neurocritical care staff who tirelessly expose themselves daily and knowingly to traumas that affect not only their own health, but also the quality of care they provide to patients and families.

\section{Conflict of interest}

Ana-Maria Vranceanu declares that she has no conflict of interest.

\section{Publisher's Note}

Springer Nature remains neutral with regard to jurisdictional claims in published maps and institutional affiliations.

Published online: 13 August 2019

\section{References}

1. Caregiving in the U.S. 2015 | National Alliance for Caregiving. National Alliance for Caregiving https://www.caregiving.org/caregiving2015/. Accessed 24 Dec 2018.

2. Shaffer KM, Riklin E, Stagl JM, Rosand J, Vranceanu AM. Mindfulness and coping are inversely related to psychiatric symptoms in patients and informal caregivers in the neuroscience ICU: implications for clinical care. Crit Care Med. 2016;44:2036-8 [PMID: 27513536]

3. Choi KW, Shaffer KM, Zale EL, Funes CJ, Koenen KC, Tehan T, Rosand J, Vranceanu AM. Early risk and resiliency factors predict chronic posttraumatic stress disorder in caregivers of patients admitted to a neuroscience ICU. Crit Care Med. 2018;46(713-319):29384786.

4. Purvis T, Saylor D, Burnout and resilience among neurosciences critical care unit staff. Neurocritical Care. 2019.

5. Ghawadra SF, Abdullah KL, Choo WY, Phang CK. Mindfulness-based stress reduction for psychological distress among nurses: a systematic review. J Clin Nurs. 2019. https://doi.org/10.1111/jocn.14987 [Epub ahead of print]. 